\title{
A case of pneumatosis cystoides intestinalis in a patient with Crohn's disease complicated by systemic sclerosis
}

\author{
Tyler Berger*1, Keith Shenberger ${ }^{2}$ \\ ${ }^{1}$ Gesinger Commonwealth School of Medicine, United States \\ ${ }^{2}$ UPMC Susquehanna Bone and Joint Institute, United States
}

Received: February 27, 2017

DOI: $10.5430 /$ crim.v4n3p1
Accepted: May 11, 2017

URL: https://doi.org/10.5430/crim.v4n3p1

\begin{abstract}
Pneumatosis cystoides intestinalis (PCI) is the rare clinical finding of gas accumulation within the gastrointestinal wall. It can be secondary to a variety of disease processes including Crohn's disease and systemic sclerosis. The present case describes PCI associated with pneumoperitoneum and ascites as the initial presentation of systemic sclerosis in a 64-year-old female with a history of Crohn's disease. The diagnosis of systemic sclerosis was delayed as PCI was initially attributed Crohn's disease. This report reviews the pathogenesis, clinical presentation, and management of PCI in relation to both diseases.
\end{abstract}

Key Words: Colorectal surgery, Pneumatosis cystoides intestinalis, Gastroenterology, General surgery, Crohn's disease, Systemic sclerosis

\section{INTRODUCTION}

Systemic sclerosis ( $\mathrm{SSc}$ ) is a collagen vascular disease that causes inflammation and fibrosis of multiple organ systems. Cutaneous sclerosis is a universal finding. ${ }^{[1,2]}$ Other commonly observed dermatological features include calcinosis, telangiectasia, and pigmentation changes. ${ }^{[1]}$ Vasomotor instability as manifested by Raynaud's phenomenon is another characteristic finding. ${ }^{[1,2]}$ SSc may involve a variety of extracutaneous organs with the most frequent being the urinary, pulmonary, and gastrointestinal systems. ${ }^{[2]}$ Renal and pulmonary manifestations result in the highest morbidity; however, gastrointestinal disease is more prevalent and present in $70 \%-98.9 \%$ of SSc patients. ${ }^{[2]}$ The most common gastrointestinal complications include gastroesophageal reflux, gastritis, intestinal dysmotility, pseudo-obstruction, chronic diarrhea, and fecal incontinence. ${ }^{[2]}$ While gastrointestinal disease is frequent, pneumatosis cystoides intestinalis (PCI) is an extremely rare manifestation of SSc gastrointestinal involvement. ${ }^{[2]}$

PCI, a clinical finding of gas within the gastrointestinal wall, presents most often as an incidental finding on imaging. ${ }^{[3]}$ A literature review of $39 \mathrm{PCI}$ cases with underlying SSc noted that the presentation of PCI occurred at a median age of 57 years and after a mean disease duration of 6 years. ${ }^{[4]}$ PCI can be secondary to numerous etiologies other than SSc including diseases of the pulmonary system, immunodeficiency, immunosuppressive therapy, organ transplantation, infection, iatrogenic causes, other connective tissue disease and gastrointestinal disease including Crohn's disease. ${ }^{[3,5-7]}$ Approximately $15 \%$ of cases are primary which represents idiopathic etiology. ${ }^{[5]}$

The diagnosis of the underlying etiology in secondary PCI can be difficult due to the broad differential diagnosis and, occasionally, the presence of multiple known disease associ-

*Correspondence: Tyler Berger; Email: berger@tcmc.edu; Address: 1201 Northway Rd Apartment 1202 Williamsport PA 17701, United States. 
ations within a single case. ${ }^{[8]}$ Furthermore, the decision of management is often challenging as PCI creates a clinical picture with the appearance of a surgical abdomen despite typically representing a benign process. ${ }^{[9,10]}$ The presence of ascites and pneumoperitoneum may further complicate the determination of appropriate management. ${ }^{[9]}$ The case presented here describes PCI associated with pneumoperitoneum and ascites as the initial presentation of SSc in a 64-year-old female with history of Crohn's disease. PCI was successfully managed conservatively; however, the diagnosis of SSc was delayed as PCI was initially attributed to Crohn's disease alone. The pathogenesis, clinical manifestations, and management of PCI with relation to SSc and Crohn's disease are discussed in this report.

\section{Case presentation}

A 64-year-old female with a history of Crohn's disease presented to her primary care physician with mild episodic abdominal pain, diarrhea and bloating of several weeks duration. The patient's Crohn's disease is in remission for the past 5 years on sulfasalazine therapy. Her last bowel movement was the day prior to the visit. Exam revealed severe abdominal distention. Radiographs demonstrated dilated large and small bowel loops with free intra-abdominal air. She was referred to the emergency room. Assessment there revealed no additional complaints. On exam she was nontoxic in appearance. Her vitals were as follows: temperature $37^{\circ} \mathrm{C}$, pulse $84 \mathrm{bpm}$, respiratory rate 18 , and blood pressure 110/63. Abdominal examination was remarkable for moderate abdominal distention but tenderness to palpation and signs of peritoneal irritation were notably absent. The patient's abdomen was tympanic with normoactive bowel sounds and no masses were noted. Computed tomography (CT) revealed a large amount of free intra-abdominal air, small colonic cystic air accumulations, and free fluid accumulation in the left colic gutter and posterior cul-de-sac (see Figure 1). The small and large bowels were markedly dilated without evidence of obstruction. Laboratory analysis was reassuring with a WBC count of 6,900 with a normal differential, PT 10.2 seconds, PTT 28 seconds, albumin $4.2 \mathrm{~g} / \mathrm{dl}$, AST $22 \mathrm{IU} / \mathrm{L}$, ALT $23 \mathrm{IU} / \mathrm{L}, \mathrm{CO}_{2} 24.9 \mathrm{mmol}$, anion gap 10, creatinine $0.91 \mathrm{mg} / \mathrm{dl}$, and BUN $16 \mathrm{mg} / \mathrm{dl}$. Based on imaging and her clinical context, the free air was attributed to PCI. Given that she was relatively asymptomatic and signs of peritoneal irritation were absent, she was discharged and advised to remain on clear liquids for the remainder of the day. Three months later, surgical referral was made due to persistent abdominal distention. A CT enhanced with contrast redemonstrated pneumoperitoneum, free abdominal fluid and dilation of large and small bowel loops (see Figure 2). Surgery was deferred as she remained relatively asymptomatic except for persistent diarrhea and abdominal distention. These symptoms resolved two months after the surgery consult.

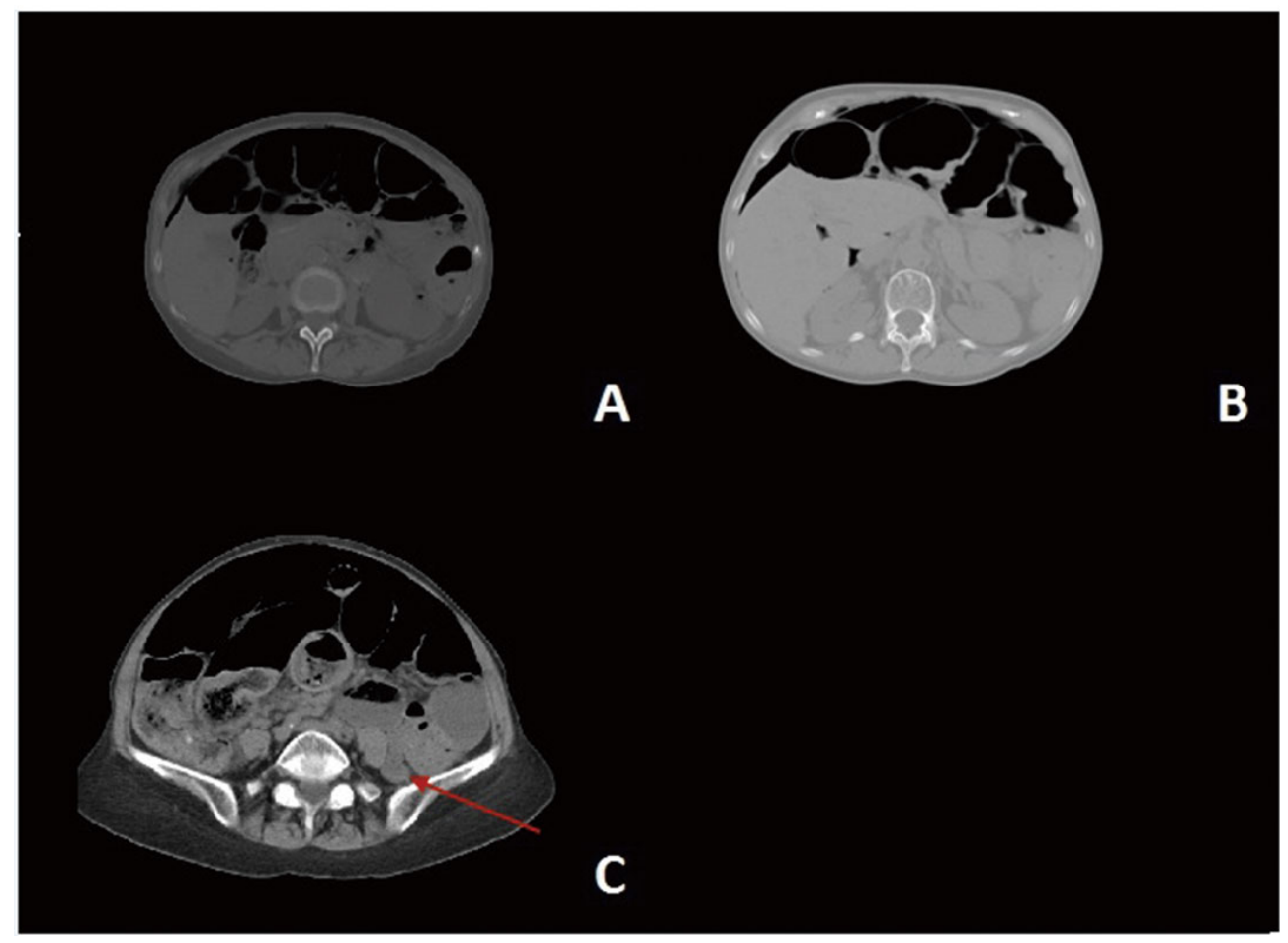

Figure 1. Abdominal computed tomography axial views at presentation to emergency room. A) Intramural air accumulations within the bowel wall. B) Pneumoperitoneum. C) Red arrow notes free fluid accumulation in left colic gutter. 


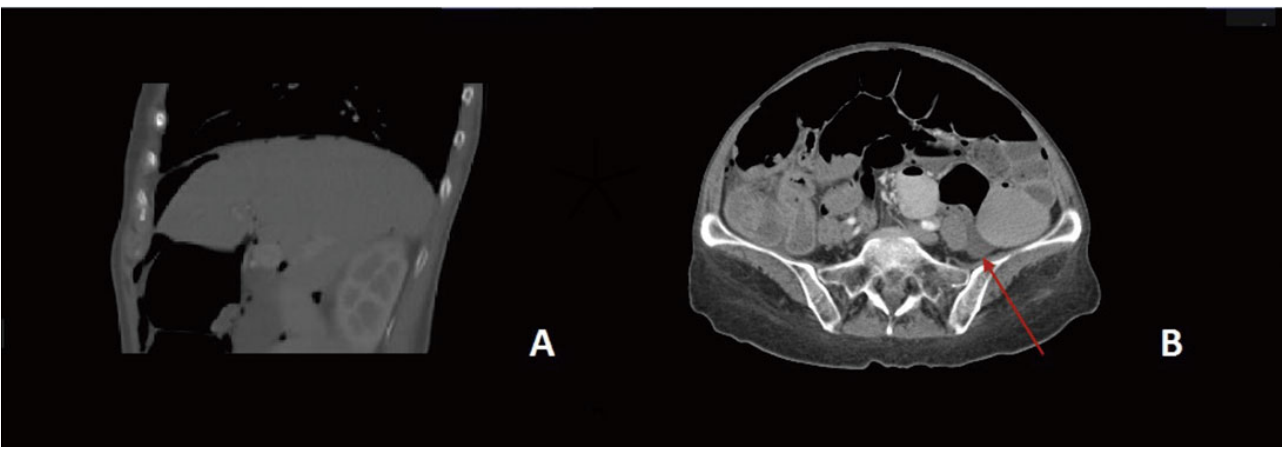

Figure 2. Contrast enhanced abdominal computed tomography 3 months after PCI presentation. A) Sagittal view demonstrating pneumoperitoneum. B) Axial view with the red arrow noting free fluid accumulation in left colic gutter.

During this time, she developed Raynaud's phenomenon, progressive gastroesophageal reflux and dysphagia. Upper gastrointestinal series revealed mild thickening of the distal esophagus. Subsequent evaluation by rheumatology was remarkable for skin thickening extending just proximal to the metacarpophalangeal joints bilaterally. Telangiectasia and calcinosis were notably absent. Serological testing yielded an ANA greater than 1:1,280 with negative anti-Scl-70 and anti-centromere antibodies. A creatinine of $1.38 \mathrm{mg} / \mathrm{dl}$ and
BUN of $18 \mathrm{mg} / \mathrm{dl}$ with an unremarkable chest X-ray were observed. Further questioning did not yield any pulmonary complaints. In light of skin involvement extending beyond the bilateral metacarpophalangeal joints, the diagnosis of SSc was made. Eight months after the discovery of PCI, a follow-up enhanced CT demonstrated mild bowel dilation with resolution of PCI, pneumoperitoneum and peritoneal fluid (see Figure 3).

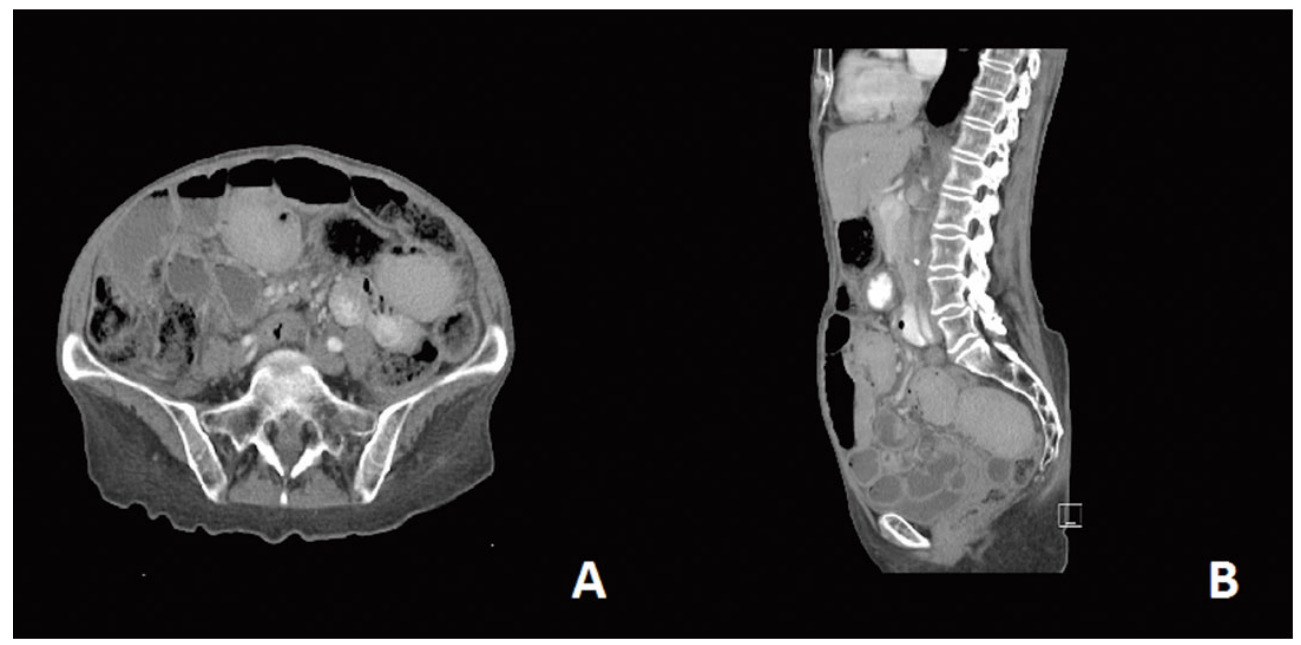

Figure 3. Contrast enhanced abdominal computed tomography 8 months after presentation of pneumatosis cystoides intestinalis demonstrating mild small and large bowel dilation with absence of pneumonitis intestinalis, free fluid and pneumoperitoneum in A) axial and B) sagittal views.

\section{Discussion}

PCI is a rare clinical finding of cystic gas accumulations within the bowel wall. PCI can be secondary to variety of disorders, including Crohn's disease and SSc. The present case describes PCI as the initial presentation of SSc which has been reported in one case report previously. ${ }^{[11]}$ However, the concurrent history of Crohn's disease is an additional contribution to the generation of PCI in this case and resulted in a delayed SSc diagnosis. The present case highlights two distinct diseases processes that contribute to the pathogenesis of PCI.

However, regardless of the underlying cause, the pathogenesis of PCI can be best characteristic by the gas source. ${ }^{[5]}$ Three gas sources have been implicated in PCI: pulmonary, intraluminal and bacterial. ${ }^{[5]}$ Increased intestinal pressure is thought to mediate intraluminal gas accumulation in the bowel wall by elevating gas partial pressure in the gut lumen. ${ }^{[5,8]}$ As a result, a diffusion gradient is created for gas 
to enter the bowel wall. Likewise, excess bacterial gas production in intestinal lumen is thought to create a diffusion gradient in a similar manner. ${ }^{[5]}$ Interestingly, pulmonary gas can be a source for bowel wall air accumulations. ${ }^{[5,8]}$ It has been suggested that when alveoli are damaged, gas travels along blood vessels, into the mesentery and then causes subserosal cysts. ${ }^{[5]}$

Damage to the mucosal barrier is thought to mediate the development of PCI in Crohn's disease. ${ }^{[5]}$ Inappropriate activation of a Th1 and Th17 mediated immune response produces an inflammatory infiltrate within the gut wall. ${ }^{[12]}$ As a consequence, the mucosal lining of intestinal tract is damaged. ${ }^{[12]}$ The loss of gut mucosa increases the permeability of the bowel wall to bacterial gas that is normally localized to the lumen. ${ }^{[5]}$ The patient in the present case is in 5 years remission at the onset of PCI. However, even after disease control with immunosuppressive therapy, mucosal damage persists in the majority of patients with Crohn's disease. ${ }^{[12]}$

The pathogenesis of PCI in relation to SSc is unknown; however, it is thought that intestinal hypomotility and subsequent pseudo-obstruction plays a prominent role in the majority of cases. ${ }^{[2,13]}$ Intestinal dysmotility has been observed $44 \%$ - $88 \%$ of patients with SSc. ${ }^{[2]}$ Several factors contribute to intestinal hypomotility. Early in the disease process, reduced expression of vascular endothelial growth factor impairs angiogenesis leading to decreased vascular supply of the intestinal choroid plexus and smooth muscle. ${ }^{[2]}$ Likewise, smooth muscle collagen deposition in later disease compresses the neurovascular supply to the gastrointestinal tract, further compromising bowel vascular supply and innervation. ${ }^{[2]}$ Anti-muscarinic antibodies have also been implicated in bowel hypomotility secondary to SSc. ${ }^{[2]}$ Ultimately, marked reduction in gastrointestinal motility is the underlying etiology of pseudo-obstruction in $\mathrm{SSc}^{[2]}$ and is thought to mediate the development of PCI in this population. ${ }^{[2,6,13,14]}$ Although the pulmonary manifestations of SSc are clinically absent from this case, it is well known interstitial lung disease and pulmonary vascular disease are complications of the disease. ${ }^{[1,2]}$ As a result, pulmonary involvement may represent an additional contribution to PCI development in SSc.

In this patient, SSc and Crohn's disease may have each uniquely contributed to the etiology of PCI. Longstanding damage from Crohn disease may have decreased the barrier function of the mucosal surface. Upon development of SSc, a pseudo-obstruction occurred as demonstrated by significant dilation of bowel loops on abdominal CT without evidence of a mechanical obstruction. Pseudo-obstruction increases intraluminal pressure, ${ }^{[5]}$ which in conjunction with Crohn's induced mucosal injury, may have been the inciting event that led to gas accumulation within the gut wall. While atypical, a prior case report has described pseudo-obstruction as the initial presentation of SSc. ${ }^{[14]}$

Clinically, most cases of PCI are asymptomatic at presentation. ${ }^{[3,6,7]}$ Of those that are symptomatic, the most common symptoms are diarrhea, bloody stool, abdominal pain, distention, constipation, and weight loss. ${ }^{[3,6,8]}$ Physical examination is most often unrevealing, however $29 \%$ of cases have signs of an acute abdomen. ${ }^{[6]}$ Secondary PCI often does not create signs suggestive of the underlying etiology; ${ }^{[8]}$ however, pseudo-obstruction on imaging is indicative of SSc. ${ }^{[3,6,13-15]}$ Imaging may also reveal pneumoperitoneum and rarely ascites associated with PCI. ${ }^{[5-9]}$ Pneumoperitoneum is thought be a consequence of subserosal bleb rupture leading to intraabdominal air accumulation without true transmural perforation of the gut wall. ${ }^{[5,6]}$ A prior case report has described both features with underlying Crohn's disease. ${ }^{[7]}$ The presence of both pneumoperitoneum and ascites complicates the clinical picture and raises the question for the need of surgical intervention. ${ }^{[8]}$

Review of the literature yielded mixed results with regard to the management of PCI associated with either Crohn's or SSc. PCI associated with SSc has been managed both surgically ${ }^{[10,15,16]}$ and conservatively. ${ }^{[4,11,14,17]}$ Likewise, conservative $^{[3,18]}$ and surgical management ${ }^{[7,19-21]}$ has been reported in PCI with underlying Crohn's disease. Determining the need for surgical intervention is perhaps the most important decision regarding PCI treatment. ${ }^{[3,7,8]}$ There are several important considerations that direct the need for surgery in PCI cases associated with either disease. First, the finding of portal venous gas should raise concern as it may indicate mesenteric infarction and a poor prognosis. ${ }^{[3,6-9,21]}$ Prospective studies have demonstrated a $37 \%$ mortality rate in these patients. ${ }^{[7]}$ Likewise, patients with laboratory valves suggestive of an underlying life threatening etiology including a lactate of greater than $2 \mathrm{mmol}$, bicarbonate of less than $20 \mathrm{mEq} / \mathrm{L}$, amylase of more than $200 \mathrm{U} / \mathrm{L}$, pH of less than 7.3 or signs of disseminated intravascular coagulation should undergo emergent surgery. ${ }^{[6]}$ Last, signs of an acute abdomen on examination or evidence of ischemia on imaging are indications for surgical intervention. ${ }^{[8]}$

As in the present case, PCI without these features may be managed conservatively. ${ }^{[8]}$ Asymptomatic PCI can be monitored closely for complications and requires no medical treatment. ${ }^{[8]}$ For symptomatic patients without signs of peritoneal irritation, several treatment modalities have yielded successful results including bowel rest, bowel decompression, antibiotic treatment, and high concentration or hyper- 
baric oxygen therapy. ${ }^{[3,6,8]}$

Last, the current case highlights a valuable teaching point. The diagnosis of systemic sclerosis was delayed for several months as the development of PCI was solely attributed to the patient's history of Crohn's disease. However, PCI with pseudo-obstruction creates a clinical picture highly suggestive of SSc. These findings should have prompted a thorough evaluation for SSc once an acute abdomen was excluded. PCI contains a broad differential of secondary disease processes and the prior history of one associated etiology should not prematurely conclude further evaluation, especially when signs of a concurrent underlying disease are present.

\section{Conclusion}

In summary, SSc and Crohn's disease each uniquely contribute to the pathogenesis of PCI. Mucosal injury mediates bowel wall air accumulation in Crohn disease. Hypomotility and pseudo-obstruction are thought to be the underlying pathological process of PCI associated with SSc. While signs and symptoms rarely suggest a secondary cause of PCI, the finding of a pseudo-obstruction is suggestive of SSc. Pneumoperitoneum and ascites may rarely complicate PCI and create the appearance of a perforated viscus. However, the determination of management must include the entire clinical context of the patient and not findings on imaging alone. Due to the broad differential of secondary PCI, even cases with a history of a known association with PCI should proceed with further evaluation for additional underlying disease processes once a surgical abdomen is ruled out.

\section{ACKNOWLEDGeMENTS}

The authors would like to thank Keith Shenberger, M.D for granting access to his clinic and Margaret Shoemaker, M.D for her editorial review of this case report.

\section{CONFLicts OF INTEREST Disclosure}

The authors have declared no conflicts of interest.

\section{REFERENCES}

[1] Viswanath V, Phiske M, Gopalani V. Systemic sclerosis: Current concepts in pathogenesis and therapeutic aspects of dermatological manifestations. Indian Journal of Dermatology. 2013; 58(4): 255. https://doi.org/10.4103/0019-5154.113930

[2] Tian X, Zhang X. Gastrointestinal Complications of Systemic Sclerosis. World Journal of Gastroenterology. 2013; 19(41). https: //doi.org/10.3748/wjg.v19.i41.7062

[3] Zhang H, Jun SL, Brennan TV. Pneumatosis Intestinalis: Not Always a Surgical Indication. Case Reports in Surgery. 2012: 1-3. PMid:23198249. https://doi.org/10.1155/2012/719713

[4] Kaneko M, Sasaki S, Teruya S, et al. Pneumatosis Cystoides Intestinalis in Patients with Systemic Sclerosis: A Case Report and Review of 39 Japanese Cases. Case Reports in Gastrointestinal Medicine. 2016. https://doi.org/10.1155/2016/2474515

[5] Peter SDS, Abbas MA, Kelly KA. The Spectrum of Pneumatosis Intestinalis. Archives of Surgery. 2003 Jan; 138(1): 68-75. PMid:12511155. https://doi.org/10.1001/archsurg. 138.1 .68

[6] Feuerstein JD, White N, Berzin TM. Pneumatosis Intestinalis With a Focus on Hyperbaric Oxygen Therapy. Mayo Clinic Proceedings. 2014 May; 89(5): 697-703. PMid:24797647. https://doi.org/ 10.1016/j . mayocp. 2014.01.026

[7] Sakurai Y, Hikich M, Isogaki J, et al. Pneumatosis Cystoides Intestinalis Associated with Massive Free Air Mimicking Perforated Diffuse Peritonitis. World Journal of Gastroenterology. 2008 Nov; 14(43). https://doi.org/10.3748/wjg. 14.6753

[8] Khalil P, Huber-Wagner S, Ladurner R, et al. Natural history, Clinical Pattern, and Surgical Considerations of Pneumatosis Intestinalis. Eur J Med Res European Journal of Medical Research. 2009 Jun; 14(6): 231. https://doi.org/10.1186/2047-783X-14-6-231

[9] Ho LM, Paulson EK, Thompson WM. Pneumatosis Intestinalis in the Adult: Benign to Life-Threatening Causes. American Journal of Roentgenology. 2007 Jun; 188(6): 1604-13. PMid:17515383. https://doi.org/10.2214/AJR.06.1309

Published by Sciedu Press
[10] Ishikawa M, Okada J, Kondo H. Five Cases of Systemic Sclerosis with associated with Intestinal Pseudo-Obstruction. Ryumachi. 1999; 39(5).

[11] Ejtehad F, Chatzizacharias NA, Kennedy H. Pneumatosis Intestinalis as the Initial Presentation of Systemic Sclerosis: A Case Report and Review of the Literature. Case Reports in Medicine. 2012. https://doi.org/10.1155/2012/987410

[12] Sartor RB. Mechanisms of Disease: Pathogenesis of Crohn's Disease and Ulcerative Colitis. Nature Clinical Practice Gastroenterology \& Hepatology. 2006; 3(7). https ://doi .org/10.1038/ncpgasth ep0528

[13] Ishikawa M, Okada J, Kondo H. Five Cases of Systemic Sclerosis with associated with Intestinal Pseudo-Obstruction. Ryumachi. 1999; 39(5).

[14] Ortiz-Alvarez O, Cabral D, Prendiville JS, et al. Intestinal PseudoObstruction as an Initial Presentation of Systemic Sclerosis in Two Children. Oxford Rheumatology. 1997; 36(2). https ://doi.org/ 10.1093/rheumatology/36.2.280

[15] Seket B, Kaczmarek D, Tiffet O. Intestinal Pseudo-Obstruction and Pneumatosis Cystoides Intestinalis in a Scleroderma Patient. Journal of the American College of Surgeons. 2007; 205(1): 180-181. https://doi.org/10.1016/j.jamcollsurg. 2006.08.025

[16] Aoyama Y. A Case Report of Acute Peritonitis with Systemic Sclerosis, Complicated by Pneumatosis Cystoides Intestinalis and Pneumoperitoneum. Journal of Japanese College of Surgeons. 2009; 34(5). http://doi.org/10.4030/jjcs.34.856

[17] Quiroz E, Flannery M, Martinez E, et al. Pneumatosis Cystoides Intestinalis in Progressive Systemic Sclerosis: A Case Report and Literature Review. The American Journal of the Medical Sciences. 1995; 6(310)

[18] Pais TP, Pinho R, Carvalho J. Hepatic Portal Venous Gas and Intestinal Pneumatosis as Initial Presentation of Crohn's Disease: First case report. Journal of Crohn's and Colitis. 2014; 8(10): 1329-1330. https://doi.org/10.1016/j.crohns.2014.02.023 
[19] Gelfond D, Blanchard SS, Malkani A. Pneumatosis Intestinalis: A Rare Presentation of Crohn Disease Exacerbation. Journal of Pediatric Gastroenterology and Nutrition. 2011; 52(2): 225-226. https://doi.org/10.1097/mpg.0b013e3181eb686c

[20] Brunton I, Hayee B, Cook A, et al. An Unexpected Cause of Small Intestinal Obstruction in Crohn's Disease. The Internet Journal of
Surgery. 2006; 10(1).

[21] Hokama A, Nagahama M, Kishimoto K, et al. Pneumatosis Intestinalis and Hepatic Portal Venous Gas in Crohn's Disease. Journal of Crohn's and Colitis. 2009; 3(4). https://doi.org/10.1016/j . crohns.2009.07.004 